J. Math. Biology (1981) 13: 23-45

\title{
Approximate Solution of a Model of Biological Immune Responses Incorporating Delay
}

\author{
A. C. Fowler* \\ School of Mathematics, 39, Trinity College, Dublin 2, Ireland
}

\begin{abstract}
A model of the humoral immune response, proposed by Dibrov, Livshits and Volkenstein (1977b), in which the antibody production by a constant target cell population depends on the antigenic stimulation at earlier times, is considered from an analytic standpoint. A method of approximation based on a consideration of the asymptotic limit of "large" delay in the antibody response is shown to be applicable, and to give results similar to those obtained numerically by the above authors. The relevance of this type of approximation to other systems exhibiting "outbreak" phenomena is discussed.
\end{abstract}

Key words: Immune response - Delay - Model

\section{Introduction}

There are many biological and other systems whose description in terms of mathematical models naturally includes one or more delays. Such delays often arise (for example in population dynamics) because of the existence of a finite maturation or gestation time, or because of finite transport time between different components which interact reciprocally (i.e. exhibit feedback). Examples of such systems in physiology are hematopoiesis and respiration, where the delays are due respectively to cell maturation and blood transport between lung and brain (Mackey and Glass, 1977).

Although introduction of a delay into a set of differential equations greatly complicates their analysis, there has been much interest in such functional equations because of the fact that a sufficiently large delay can often destabilize an otherwise stable steady state, and this in many cases leads to a stable large scale oscillation of the system. In possibly the simplest model of saturation-limited growth (the logistic equation), Jones $(1962 \mathrm{a}, \mathrm{b})$ showed that when the delay exceeded $\pi / 2$ (in appropriate units), the non-trivial steady state became unstable, and the population variable underwent a pronounced oscillation. Numerical integration of the equation showed that the oscillation had a definite "dormant" phase, in which the population remained at very low levels for some time before

* Present address: Department of Mathematics, Massachusetts Institute of Technology, Cambridge, MA 02139, USA 
rising and falling comparatively rapidly in a spike-shaped pulse. The form of this spiky oscillation is felt to be due to the presence of a substantial delay in the equation.

More realistic models, for example in population dynamics, incorporate a distributed delay or age structure (e.g. Cushing, 1977; Rubinow, 1978) which may reflect the fact that maturation time varies from individual to individual. Typically, one replaces a delayed term $y(t-\tau)$ by an integral of the form $\int_{0}^{\infty} K(\tau) y(t-\tau) d \tau$, where $K(\tau)$ is the delay kernel. A discrete delay results from taking $K(\tau) \propto \delta\left(\tau-\tau_{0}\right)$ (i.e. the delta function). This may be a reasonable approximate formulation in cases where $K$ is distributed about a maximum at $\tau=\tau_{0}$ and falls off rapidly for $\tau \lessgtr \tau_{0}$. This seems reasonable for cell maturation delays. If this is not the case (i.e. some individuals mature almost immediately), then a delta function is not a realistic model kernel, and we must resort to other techniques. Particular kernels of the form $\tau^{n} e^{-a \tau}$ are susceptible to analysis. In this case, a differential delay equation is reducible to a set of ordinary differential equations (Macdonald, 1978), and thus there is a qualitative link between sets of such equations and delay equations.

The relevance of this becomes apparent when we realize that there are other physical phenomena which exhibit the same kind of spiky pulses, interspersed with long dormant periods of relative inactivity. We mention two: firstly, the periodic outbreak of spruce budworm larvae in the forests of New Brunswick (Ludwig et al., 1978). Secondly, the periodic outburst (jökulhlaup) of an ice-dammed lake (Grimsvötn) in Iceland (Nye, 1976). The similarity of both these phenomena to the computed periodic solution of the delayed logistic equation indicates that similar analytic approaches may be appropriate.

A further property of delay in a system is the occurrence of chaotic fluctuations (Mackey and Glass, 1977). Those authors show that a single differential delay model of hematopoiesis can exhibit chaos [a term introduced in the context of difference equations by $\mathrm{Li}$ and Yorke (1975)], which is observed in white blood cell populations of leukemia patients. Chaotic behaviour is also observed in the solution of sets of ordinary differential equations, as in the famous Lorenz model (Lorenz, 1963) which exhibits the spiky behaviour discussed above.

The complexity of the system behaviour observed in many different physical and biological systems makes it worthwhile to try and develop analytical tools for the approximate solution of relevant models, and the particular pulse-like oscillation observed in such systems suggests that some unifying physical process may govern the essential behaviour.

The purpose of the present paper is to provide such an analytic solution for a model proposed by Dibrov et al. (1977a), and analyzed by them there and in a subsequent paper (1977b). Their analysis was qualitative, whereas the present work gives a quantitative approximate solution of the equations, motivated by the form of their numerical results.

When a foreign body (antigen) is injected into an animal, it responds by producing antibodies which are able to bind with the antigen and effectively neutralize it. It is easy to visualize the interaction as a second order prey-predator system in which the antibody plays the role of predator. The immune response of antibody production is however delayed by the maturation time of the cells. Dibrov et al. (1977b) showed that this delay could destabilize the steady state solution 
of the deterministic equation, and if sufficiently large, the immune response would be incapable of coping with the rise in density of a proliferating antigen. Such an increase would be interpretable as the death of the animal (although this model does not discuss the cell-mediated response). In the intermediate range of delay, numerical integration showed that the deterministic equations had periodic solutions in the form of pronounced spiky pulses, in which three phases are evident: dormant, when both antigen and antibody are at low levels; active, when antigen rapidly increases; and controlled, when antigen rapidly decreases, and antibody suddenly increases and decreases.

It is the form of these periodic solutions (where there is a definite "flat patch") that suggests the use of an approximation method to the applied mathematician: we ask such questions as: what is the appropriate approximation procedure to adopt? How does the period vary with the delay? How does the minimum antigen density vary with the delay?

This last question is of particular importance, since the antigen population actually consists of individuals, and hence the deterministic equations become stochastic when population levels of the order of a few individuals are approached. This enables the antigen to be totally eliminated even though its deterministic measure cannot attain zero. A stochastic analysis (Dibrov et al., 1977b) shows, as we might expect, that the probability of extinction within a cycle becomes nonnegligible as the minimum antigen population decreases to $\sim 1$ individual. Rather than carry out a stochastic analysis, we will define this population size as a cut-off level, above which there is a strong possibility of a relapse of the infection. Since we shall find that the minimum depends very sensitively on the delay, this effectively enables us to define a precise "transition" delay above which antigen elimination is virtually certain, and hence the possibility of relapse very small. It would seem that the application of this analysis would be relevant to the study of recurrent epidemics.

Experimental evidence for cyclic antibody production is surveyed in the article by Weigle (1975): see also the papers by Romball and Weigle (1973) and Tew and Mandel (1978). The experimental evidence these authors describe indicate that the results of the present paper [and those of Dibrov et al. $(1977 \mathrm{a}, \mathrm{b})]$ may be of genuine relevance to immunology.

Approximate solutions for ordinary differential equations often rely on the presence of a small parameter, which may be used to obtain an asymptotic representation of the solution. When a delay is present, this is not easily done, and other methods have been sought. Macdonald (1978) considers particular delay kernels (as described above) such that a single differential delay equation becomes a set of ordinary differential equations. Bifurcation theory can be applied to the question of the existence of small oscillations, but analytic solutions seem no nearer at hand. A slightly less artificial method is to obtain a sequence of differential equations by splitting a discrete delay interval $(t-\tau, t)$ into a set of small subintervals $(t-(n+1) h, t-n h), n=0,1, \ldots, N-1, N h=\tau$. Defining variables $y_{n}(t)=y(t-n h)$, and using $y_{n+1}(t)-y_{n}(t) \approx h y_{n}^{\prime}(t)$, again yields a system of $N$ approximating ordinary differential equations (Banks, 1977). The approximation tends to the delay equation as $N \rightarrow \infty$ : no apparent simplification is made to the problem of obtaining an analytic solution. 
Morris (1976) addressed the problem of obtaining the form of the periodic solution of the delayed logistic equation when the delay is marginally supercritical (i.e. the non-trivial steady state is weakly unstable). He used the Poincare-Lindstedt method of straining the time variable.

In the present work, we seek to obtain analytic forms for oscillating solutions in immune processes which have a characteristic spiky behaviour interspersed with dormant periods. This characteristic inactive phase of the oscillation immediately suggests some kind of asymptotic limiting behaviour, and we here adopt the notion that the delay is "large" (in a sense to be made precise in due course). The singular limit in which the delay tends to infinity was studied analytically by Fowler (1981) for the delayed logistic equation. The result of the analysis was that (normalized) values of the delay of 2 gave surprisingly accurate results and that 3 could certainly be classed as a very large delay. Moreover, values of the minimum, and of the period, depended very sensitively on the delay, and thus this method of analysis seemed well vindicated by the results.

In this paper we complement the work of Dibrov et al. (1977b) by extending the quantitative analysis of the delayed logistic equation, described above, to their model of antigen-antibody interaction in the humoral immune response. In the next section, we describe the nature of this response and its formulation as a mathematical model, and summarize the known results concerning this model. In $\S \S 3$ and 4 we carry out a detailed analytical approximation based on the method of matched asymptotics (Cole, 1968). The interested immunologist can profitably skip these sections, since the main results are restated in $\$ 5$, together with a comparison of the results with Dibrov's et al. (1977b) numerical computations, and a discussion of their significance.

\section{Mathematical Model}

Before describing the model due to Dibrov et al. (1977b), we first describe the essential nature of the mammalian immune response, based on the clonal selection theory of Burnet, which is now generally accepted. The nature of this response is described in the books by Eisen (1974) and Hood et al. (1978), and brief summaries are given by Bell (1970) and Dibrov et al. (1977a). For completeness, we give also a brief resumé here.

The immune system operates by virtue of the response of certain types of cell to the presence of a foreign organism, or antigen. There are two types of response. The cell-mediated response is particularly stimulated by fungi and parasites, and is partly due to the presence of "scavenging" cells such as granulocytes and macrophages, but mainly occurs by activation of $T$-cells (see below), which eliminate the invading cells by combining directly with them, and (for example) lysing. Thus the interaction is direct, and there is no specificity. The humoral response, with which we shall be entirely concerned, occurs through the response of other types of cells called $B$-lymphocytes to antigenic contact, particularly with bacteria: it is this response which Burnet's clonal selection theory deals with.

If an antigen is administered to an animal, it responds by producing quantities of antibodies, which can combine with the antigen and thus effect its elimination from the system. This primary response is often slow (taking about a week). 
Subsequent injections of the antigen, however, lead to a much more rapid production of antibodies and hence also elimination of the antigen: this fact is the basis of immunization procedures.

The clonal selection theory explains these facts as follows. The lymphocyte population (about $10^{12}$ cells in man) consists of two types, $T$ cells and $B$ cells. $T$ cells, so called because they arise through differentiation of stem cells in the thymus, act in a regulatory manner on the humoral response, are not central to the discussion (or the model), and will not be considered further. $B$ cells arise through differentiation of stem cells in the Bursa of Fabricius in birds or associated lymphoid tissue in mammals. The $B$ cells constitute a heterogeneous population. When any given antigen is first administered, only a small fraction [perhaps $10^{-5}$, Bell (1970)] of lymphocytes can respond to its stimulation. In this state, these are called small $B$ lymphocytes. When stimulated, these cells increase in size and acquire the ability to proliferate after a maturation period of perhaps a day. They then develop into memory cells, which will be assumed identical to small $B$ lymphocytes (i.e., clones), or plasma cells, which secrete antibody. Substantial numbers of plasma cells do not appear until about four days after the original stimulation. Large lymphocytes also secrete small amounts of antibody. It is the presence of substantial quantities of memory cells which enables an animal to produce a rapid and increased antibody response after subsequent injections with the same antigen.

The above description is of course very truncated, but should give an adequate summary of the process for the purpose of understanding the model. Mathematical models of the humoral immune response were proposed by Bell $(1970,1971)$ and a simpler but more tractable one presented by the same author (Bell, 1973). Numerical analyses of these models were performed by the author, who obtained qualitatively realistic results concerning primary and secondary response.

Perelson et al. $(1976,1978)$ analyzed a model for the humoral immune response from the point of view of determining whether natural selection had acted to produce an optimal strategy. Pimbley $(1974,1977$, and in other papers) analyzed a third order generalization of Bell's (1973) equations, seeking periodic behaviour in the neighbourhood of a critical value of an appropriate bifurcation parameter. A similar analysis is that of Merrill $(1978 \mathrm{a}, \mathrm{b})$; Waltman and Butz (1977) considered a threshold model of antibody production, which led to delays in the system.

Apart from this last, the other models cited above do not incorporate a delay due to maturation of the large $B$ lymphocytes. As pointed out by Dibrov et al. (1977a), however, such a delay ( $\sim 4$ days) is not negligible, and in view of comments in $§ 1$, might be expected to have an important effect on the dynamics of the interacting antibody-antigen populations. In particular, a proliferating bacterial colony will induce an increasing antibody population. When the bacteria start to be removed, the antibody population continues to increase, and this delayed increase can lead (as shown by Dibrov et al., 1977a, b) to the decrease of antigen to very low levels, at which more realistic stochastic processes can effect complete antigen elimination. This is essentially the case if the ratio of the delay to the bacterial doubling time is sufficiently high (but not too high, otherwise the immune response is too late to prevent indefinite increase of the antigen.

Dibrov's et al. (1977a) model consists of three coupled equations for the antibody quantity $a$, the antigen quantity $g$, and the small $B$ cell population $x$. If 
the secondary and subsequent responses to an antigen injection are similar, it is reasonable to suppose that the small $B$ cell population is relatively unaffected by these later injections, and a discussion of $x$ is only relevant to the primary and secondary response. If this is so, it is reasonable to put $x \approx$ constant, and consider the second-order set of equations describing antigen-antibody interactions,

$$
\begin{aligned}
& \frac{d g}{d t}=K g-Q a g, \\
& \frac{d a}{d t}=A H(t-T) g(t-T)-R a g-E a .
\end{aligned}
$$

Here $H(t)$ is the Heaviside step function

$$
\begin{aligned}
& H(t)=0, \quad t<0, \\
& H(t)=1, \quad t \geqslant 0 .
\end{aligned}
$$

$K, Q, A, R, E$ are rate constants. To complete (1.1) and (1.2) we require two initial conditions

$$
a(0)=0, \quad g(0)=g_{0}
$$

corresponding to an initial injection of antigen. The terms are interpretable as follows. $K$ is the overall growth rate of the antigen. We suppose this includes also cell death by, for example, granulocytic scavenging, as well as microbial growth (for bacteria). For such infectious organisms we expect $K>0$.

When antigen combines with antibody, the resulting complex can be eliminated from the system. Thus the antibody-antigen interaction results in a net loss of both antibody and antigen. The simplest assumption is that of the law of mass action, valid when the densities are below a saturation level, that is that the losses are proportional to the product of the antibody and antigen densities. The rate constants are $Q$ and $R$ in (1.1) and (1.2). They are not necessarily equal, since the number of binding sites available per molecule (or cell) is not necessarily the same. $E$ is the natural decay rate of antibodies. The only remaining term is that describing antibody production by the stimulated small $B$ cell population $x$. The rate of antibody production at time $t$ is supposed proportional to the rate of small $B$ cell stimulation at time $t-T$ : that is, there is a delay $T$ between stimulation of a small $B$ cell and production of plasma cells from it. This discrete delay approximates a more realistic spread in plasma cell production that would properly exist. The rate of small $B$ cell stimulation is supposed proportional to the small $B$ cell population $x$ and the antigen population $g$. Since $x$ is assumed a priori constant, this implies that antibody production at time $t$ is proportional to $g(t-T)$; the associated rate constant is then $A$ in (1.2). The Heaviside step function indicates that antibody production does not commence until a time $T$ after the initial injection.

In the case that $E$ is small, (i.e., we consider time scales much less than the mean antibody life-time, which in humans is $\sim 20$ days for IgG, $\sim 5$ days for IgM (Spiegelberg, 1974), Dibrov et al. (1977b) showed that with $K>0$, either the antigen proliferated indefinitely $(g \rightarrow \infty)$, or vanished $(g \rightarrow 0)$ as $t \rightarrow \infty$. (The case of a non-proliferating antigen is not considered here.) 
In the particular case that the humoral immune response is effective over times comparable to the lifetime of antibodies, the term $E a$ must be taken into account. In this case Dibrov et al. (1977b) found the following results: there is a non-trivial equilibrium point of (1.1) and (1.2) where $d a / d t=0$ and $d g / d t=0$ when

$$
a=a_{s t}=\frac{K}{Q}, \quad g=g_{s t}=\frac{E}{R}\left[\frac{K R / A Q}{1-(K R / A Q)}\right] .
$$

The trivial equilibrium $a=g=0$ is always linearly unstable. We suppose $K>0$, $K R / A Q<1$ (so that $g_{s t}>0$ ). Then if the delay $T$ is sufficiently small, the equilibrium point $\left(a_{s t}, g_{s t}\right)$ is stable. As $T$ is increased, it becomes linearly unstable, and a stable periodic solution bifurcates away from $\left(a_{s t}, g_{s t}\right)$ when $T>T_{1}$, where $T_{1}$ is given parametrically by

$T_{1} K=\frac{\xi}{\sin \xi}, \quad T_{1} E=\frac{[1-K R / A Q] \xi \sin \xi}{\cos \xi-(K R / A Q)}, \quad 0 \leqslant \xi \leqslant \cos ^{-1}\left(\frac{K R}{A Q}\right)$.

As $T$ increases further, this oscillation rapidly acquires the spiky characteristic discussed in $\S 1$, until for $T>T_{2}$ the periodic motion disappears altogether, and the antigen proliferates indefinitely. $T_{2}$ is given by

$$
T_{2} K=M, \quad M e^{-M}=\frac{1}{e} \frac{K R}{A Q}, \quad M>1
$$

It is the existence of a very low minimum in the oscillation which enables the antigen to be eliminated by virtue of the realistic discrete nature of the population. As already discussed, this enables us to define a critical delay $T_{c}$ by

$$
T=T_{c} \quad \text { when } \quad \min g=1 \text { cell. }
$$

Then for $T<T_{1}$, a stable antigen presence is feasible; for $T_{1}<T<T_{c}$, a stable oscillation in antigen is supportable; if $T_{c}<T<T_{2}$, the antigen will "almost certainly" be eliminated; and if $T>T_{2}$, the antigen will proliferate indefinitely. It is the oscillating parameter region $T_{1}<T<T_{2}$ that is our concern here, and we seek to establish analytic approximations by which we can evaluate $T_{c}$, $\max g$, and so on. Apart from the immune response, we suggest that these methods may be applicable to other kinds of outbreak phenomena, as discussed in $\S 1$.

In order to proceed, we make the equations dimensionless. To do this, we define

$$
g=g_{s t} g^{*}, \quad a=a_{s t} a^{*}, \quad t=T t^{*}
$$

Defining also

$$
\alpha=K T, \quad \beta=\frac{E T}{1-\kappa}, \quad \kappa=\frac{K R}{A Q},
$$

we find, dropping asterisks for convenience,

$$
\begin{aligned}
& \dot{g}=\alpha g[1-a], \\
& \dot{a}=\beta\left[g_{1}-a\{1-\kappa+\kappa g\}\right],
\end{aligned}
$$


where $g_{1} \equiv g(t-1)$, and a dot denotes differentiation. We have further put $H=1$ in (1.2), since we are not interested in the approach to the limit cycle, but rather the form of the limit cycle itself.

In dimensionless terms, the previous results are that if $\kappa<1, g_{s t}>0$; then if

$$
\kappa>\alpha e^{1-\alpha},
$$

$g \rightarrow \infty$, whereas if $\kappa<\alpha e^{1-\alpha}$, (and $T>T_{1}$, as we assume) an oscillation exists. A sufficient condition for $T>T_{1}$ is that $\alpha>\pi / 2$.

Of the six independent parameters, we see that there are only three significant dimensionless groups $\alpha, \beta, \kappa$. By analogy with Fowler (1981), the approximation of "large" delay will be sought by examining the asymptotic limit $\alpha \rightarrow \infty$. Biologically, this means that the ratio of the delay to the natural antigenic growth time becomes large. Although this may occur for a finite delay, we shall often associate the largeness of $\alpha$ with the delay, and this is what we mean by a "large" delay. If $T$ is large relative to $1 / K$, so that $K T \gg 1$, it is natural to equivalently let $\beta$ become large in such a way that $\alpha, \beta \rightarrow \infty, \alpha / \beta$ fixed; if $\alpha \rightarrow \infty$, it follows from (1.12) that the critical value of $\kappa$ above which periodic solutions do not exist tends to zero. In analyzing such solutions, we therefore expect a necessary choice of scale to be such that $\kappa \rightarrow 0$ as $\alpha \rightarrow \infty$. We shall not assume this a priori, however, but rather show that it follows naturally from the scaling of the equations: the criticality condition (1.12) will in fact emerge from the analysis, which perhaps shows the power of the method.

Apart from varying the delay, it also makes sense to vary $K$, the bacterial growth rate. Assuming that $\kappa<1$, we have $\beta / \alpha \sim E / K$, unless $\kappa \approx 1$, in which case we know $\alpha \rightarrow \infty$ cannot give a periodic solution [cf. (1.12)]. Now $E / K \sim$ doubling time of antigen divided by mean decay time of antibodies : in the immune system, the latter is of the order of 10 days. Dibrov et al. (1977b) take $E / K=3$, thus corresponding to a doubling time for antigen of 30 days, $K \sim 0.02$ day $^{-1}$. This only implies $\alpha \sim 0.1$, which is not suitable. It seems more reasonable (if $\alpha \sim 2$, say) to take $K \sim \frac{1}{2}$ day $^{-1}$, whence the doubling time is $\sim 1.4$ days.

Then $E / K \sim 0.14 \sim \beta / \alpha$, rather than $\beta / \alpha=6$. With this note of caution, we will however adopt Dibrov's et al. numerical orders of magnitudes, since it is their results we wish to elucidate, and since also we expect that parameter values may vary substantially in different contexts.

\section{Approximation Procedure}

We seek an approximate analytic form to the periodic solution of

$$
\begin{aligned}
& \dot{g}=\alpha g[1-a], \\
& \dot{a}=\beta\left[g_{1}-a\{1-\kappa+\kappa g\}\right],
\end{aligned}
$$

as $\alpha, \beta \rightarrow \infty$. The procedure followed is closely analogous to that of Fowler (1981), and we present here the main results, skipping calculational details in order to clarify the results.

Our expectation is that the oscillation (of $g$, for example) will consist of a series of pulses separated by long intervals in which both $a$ and $g$ are close to zero. In these 
regions $g$ grows exponentially, and this motivates the introduction of an exponential time variable

$$
T=e^{\alpha t} \text {. }
$$

This time variable is not to be confused with the delay in the previous section: it is used as in (2.2) only in this section. From (2.2),

$$
\frac{d}{d t}=\alpha T \frac{d}{d T}
$$

whence (2.1) becomes

$$
\begin{gathered}
T \frac{d g}{d T}=g[1-a], \\
v T \frac{d a}{d T}=g(\delta T)-a\{1-\kappa+\kappa g\},
\end{gathered}
$$

where

$$
\delta=e^{-\alpha}, \quad v=\alpha / \beta .
$$

Dibrov et al. obtain spike-like solutions for $v=\alpha / \beta=K(1-\kappa) / E=\frac{1}{6}, \alpha=1.5$ and 1.75 ; i.e., $\delta \sim \frac{1}{6}$.

We can define the time origin of $t$ so that $g \approx 1$ at $t=0$ (this is made more precise below); then in the dormant phase in $t<0$ where $g, a$ are small, we expect $g \sim T, T \lesssim 1$; this motivates the change of variable

$$
g(T)=T u(T)
$$

and so

$$
g(\delta T)=\delta T u(\delta T)
$$

With $v$ small, (2.4) $)_{2}$ suggests $a \sim \delta T$ in $T \lesssim 1$, and thus we put

$$
a=\delta T v(T)
$$

then (2.4) becomes

$$
\begin{aligned}
\frac{d u}{d T} & =-\delta u v, \\
\nu T \frac{d v}{d T} & =u(\delta T)-v\{1+v-\kappa+\kappa T u\} .
\end{aligned}
$$

The asymptotic procedure now consists of seeking series solutions in $\delta$ of (2.9) by expanding $u(\delta T)$ in a Taylor series about $T=0$ (i.e., about $t=-\infty$ ). Of course, no such series exists for the actual solution, but if the previous pulse is sufficiently far in the past (for example, at $T \sim \exp [-0(1) / \delta]$ ), then from the point of view of an asymptotic expansion in powers of $\delta$, the previous pulse is irrelevant (since $\exp [-0(1) / \delta] \ll \delta^{n}$ for any positive $\left.n\right)$ and hence the dormant region may conceptually be extended towards $T=0$. 
The $\delta$ on the right-hand side of $(2.9)_{1}$ suggests that we first rescale $T$ via

$$
T=\tilde{T} / \delta
$$

with $u, v$ now functions of $\widetilde{T},(2.9)$ is

$$
\begin{aligned}
& \dot{u}=-u v, \\
& v=\frac{u(\delta \tilde{T})-v \tilde{T} \dot{v}}{\{1+v-\kappa+\kappa \tilde{T} u / \delta\}},
\end{aligned}
$$

where a dot denotes differentiation with respect to $\tilde{T}$. Examination of $(2.11)_{2}$ now shows that in order to obtain a consistently scaled equation for $u$ in which $v \sim O(1)$, we must consider $\kappa \sim \delta$. Otherwise, $v \sim \delta, \dot{u} \ll 1$ and $g$ grows indefinitely - as in fact happens if $\kappa \sim 1$. We therefore suppose this is the case, and define

$$
\kappa=\bar{\kappa} \delta, \quad \bar{\kappa} \sim O(1) .
$$

Additionally, if $\beta / \alpha=6$, then $v \sim \frac{1}{6} \sim \delta$, if $\alpha \sim 1.5$. Although $v \ll 1$ multiplies the highest derivative of $v$, we do not expect singular behaviour since $v$ (with $v=0$ ) is then a single valued function of $u$ and $u(\delta \tilde{T})$. To make things simpler, we put $v=0$. (2.11) finally becomes the first order equation,

$$
\frac{d u}{d \tilde{T}}=-\frac{u u(\delta \widetilde{T})}{1-\bar{\kappa} \delta+\bar{\kappa} \widetilde{T} u} .
$$

The assumption of regularity of the asymptotic expansion for $u$, which also satisfies (2.13a), implies we may without loss of generality choose

$$
u(0)=1 \text {; }
$$

this simply fixes the origin of $t$.

We now solve (2.13) as a power series in $\delta$. From (2.13) we compute

$$
\dot{u}(0)=-1+O(\delta),
$$

so that

$$
u(\delta \tilde{T})=1-\delta \tilde{T}+O\left(\delta^{2}\right)
$$

hence $u$ satisfies

$$
[1-\bar{\kappa} \delta+\bar{\kappa} \widetilde{T} u] \dot{u}+[1-\delta \tilde{T}] u=O\left(\delta^{2}\right) .
$$

We seek a series expansion for the solution of (2.15) in powers of $\delta$. This is facilitated by seeking an implicit solution in the form

$$
\widetilde{T} \sim \widetilde{T}_{0}(u)+\delta \widetilde{T}_{1}(u) \cdots .
$$

Equating powers of $\delta$, and using $d u / d \tilde{T}=[d \tilde{T} / d u]^{-1}$, we find

$$
\begin{gathered}
1+\bar{\kappa} \widetilde{T}_{0} u+u \dot{\widetilde{T}}_{0}=0, \\
\dot{\tilde{T}}_{1}+\bar{\kappa} \widetilde{T}_{1}=\frac{\bar{\kappa}}{u}+\widetilde{T}_{0} \dot{\tilde{T}}_{0},
\end{gathered}
$$

etc. (a dot now denotes differentiation with respect to $u$ ), with boundary condition 
$\widetilde{T}=0$ when $u=1$, hence

$$
\widetilde{T}_{0}(1)=\widetilde{T}_{1}(1)=\cdots=0
$$

The solutions are

$$
\begin{aligned}
& \widetilde{T}_{0}(u)=e^{-\bar{\kappa} u} \int_{u}^{1} e^{\bar{\kappa} \phi} \frac{d \phi}{\phi} \\
& \widetilde{T}_{1}(u)=-e^{-\bar{\kappa} u} \int_{u}^{1}\left(\frac{\bar{\kappa}}{u}+\widetilde{T}_{0} \dot{\tilde{T}}_{0}\right) e^{\bar{\kappa} u} d u
\end{aligned}
$$

higher order terms can be computed similarly.

The above expansion becomes invalid when (2.14) is no longer asymptotic, i.e., when $\tilde{T}_{0} \sim 1 / \delta$. From (2.20), this is when $u \rightarrow 0$, and the exact asymptotic behavior as $u \rightarrow 0$ may be found by integrating by parts:

$$
\begin{aligned}
& \widetilde{T}_{0} \sim-\ln u+C+O(u \ln u), \\
& \widetilde{T}_{1} \sim \frac{1}{2} \ln ^{2} u+(\bar{\kappa}-C) \ln u+D+O\left(u \ln ^{2} u\right),
\end{aligned}
$$

with

$$
\begin{aligned}
& C=\bar{\kappa} \int_{0}^{1} e^{\bar{\kappa} u} \ln \left(\frac{1}{u}\right) d u, \\
& D=\frac{1}{2} C^{2}+\bar{\kappa} \int_{0}^{1}\left[\bar{\kappa} \ln u+\frac{1}{2} \widetilde{T}_{0}^{2}\right] e^{\bar{\kappa} u} d u .
\end{aligned}
$$

When $\tilde{T} \sim 1 / \delta, u=\exp [-O(1 / \delta)]$, and this suggests we put

$$
u(\tilde{T})=\exp [-\phi(\tilde{\tilde{T}}) / \delta], \quad \tilde{T}=\tilde{\tilde{T}} / \delta ;
$$

it follows from $(2.13 \mathrm{a})$ that when $\tilde{\tilde{T}} \sim 1, \phi$ satisfies

$$
\dot{\phi}(\tilde{T})=\frac{U(\tilde{\tilde{T}})}{1-\bar{\kappa} \delta+\mathrm{TST}} ;
$$

here a dot denotes differentiation with respect to $\widetilde{\widetilde{T}}$, and TST denotes transcendentally small terms in $\delta$ which will be neglected to all orders in an asymptotic expansion in powers of $\delta$ (Cole, 1968). Note that $U(\tilde{\tilde{T}})$ is the same function as determined implicitly by $(2.16)$ (but with $\tilde{T}$ replaced by $\tilde{\widetilde{T}}$ ), and with $u$ replaced by $U$ to distinguish the variable from the function.

(2.27) can be integrated immediately, but since $U$ is defined implicitly, it is convenient to use it as an independent variable. Thus from (2.16),

$$
\tilde{T} \sim \widetilde{T}_{0}(U)+\delta \widetilde{T}_{1}(U)+\cdots,
$$

where $\widetilde{T}_{0}, \widetilde{T}_{1}$ are given from (2.20), and (2.21). Define

$$
\phi(\tilde{\widetilde{T}})=\phi(\tilde{\widetilde{T}}(U))=\Phi(U)
$$

then (2.27) is, neglecting TST,

$$
\frac{d \phi}{d \tilde{T}}=\frac{d \Phi}{d U} / \frac{d \tilde{T}}{d U}=\frac{U}{1-\bar{\kappa} \delta},
$$


i.e.

$$
\Phi^{\prime}=\frac{U \tilde{\tilde{T}}^{\prime}(U)}{1-\bar{\kappa} \delta}=\frac{(U \tilde{\tilde{T}})^{\prime}-\tilde{\tilde{T}}}{1-\bar{\kappa} \delta}
$$

(a prime here means differentiation with respect to $U$ ). From (2.28),

$$
\Phi^{\prime} \sim \frac{\left[U\left\{\widetilde{T}_{0}+\delta \widetilde{T}_{1}+\cdots\right\}\right]^{\prime}-\left\{\widetilde{T}_{0}+\delta \widetilde{T}_{1}+\cdots\right\}}{1-\bar{\kappa} \delta} ;
$$

putting

$$
\Phi \sim \Phi_{0}+\delta \Phi_{1}+\cdots
$$

and equating powers of $\delta$ gives

$$
\begin{aligned}
& \Phi_{0}^{\prime}=\left(U \widetilde{T}_{0}\right)^{\prime}-\widetilde{T}_{0}, \\
& \Phi_{1}^{\prime}=\left(U \widetilde{T}_{1}\right)^{\prime}-\widetilde{T}_{1}+\bar{\kappa}\left[\left(U \tilde{T}_{0}\right)^{\prime}-\widetilde{T}_{0}\right],
\end{aligned}
$$

etc., with solutions

$$
\begin{aligned}
& \Phi_{0}=A+U \widetilde{T}_{0}+\int_{U}^{1} \widetilde{T}_{0} d U, \\
& \Phi_{1}=B+U \tilde{T}_{1}+\bar{\kappa} U \tilde{T}_{0}+\bar{\kappa} \int_{U}^{1} \tilde{T}_{0} d U+\int_{U}^{1} \tilde{T}_{1} d U,
\end{aligned}
$$

where $A$ and $B$ are to be determined by matching with the solutions (2.20). and (2.21). By using these latter definitions of $\widetilde{T}_{0}$ and $\widetilde{T}_{1}$ and interchanging the order of integrations, we can write (2.35) and (2.36) in the simpler form

$$
\begin{aligned}
& \Phi_{0}=A+U \tilde{T}_{0}+\frac{1}{\bar{\kappa}}\left[\tilde{T}_{0}+\ln U\right], \\
& \Phi_{1}=B+U \tilde{T}_{1}+\bar{\kappa} U \tilde{T}_{0}+\tilde{T}_{0}+\frac{1}{\bar{\kappa}} \tilde{T}_{1}-\frac{\tilde{T}_{0}^{2}}{2 \bar{\kappa}} ;
\end{aligned}
$$

here $\widetilde{T}_{0}$ and $\widetilde{T}_{1}$ are functions of $U$. Together with $\tilde{\tilde{T}} \sim \widetilde{T}_{0}+\delta \widetilde{T}_{1}+\cdots$, and $\phi=\Phi \sim \Phi_{0}+\delta \Phi_{1}+\cdots$, these equations define $\phi(\tilde{T})$ implicitly.

We now match the solutions in $\widetilde{T} \sim 1$ and $\tilde{T} \sim 1$, using the asymptotic forms of $u$ when $\widetilde{T} \gg 1$ and $\phi$ when $\widetilde{\widetilde{T}} \ll 1$. The first of these is given by (2.22) and (2.23). The behaviour of $\phi$ when $\tilde{T} \rightarrow 0$ follows by Taylor expanding $\tilde{\widetilde{T}}(U)$ given by $(2.28)$ about $U=1$ (where $\widetilde{T}=0$ ); we find

$$
\begin{aligned}
\tilde{T} \sim & (1-U)+\frac{1}{2}(1+\bar{\kappa})(1-U)^{2} \cdots \\
& +\delta\left[-\bar{\kappa}(1-U)+\frac{1}{2}\left(1-\bar{\kappa}-\bar{\kappa}^{2}\right)(1-U)^{2} \cdots\right] \cdots,
\end{aligned}
$$

and hence $\Phi$ from (2.35) and (2.36) as

$$
\Phi \sim A+(1-U)+\frac{1}{2} \bar{\kappa}(1-U)^{2}+\delta\left[B+\frac{1}{2}(1-U)^{2} \cdots\right]
$$

as $U \rightarrow 1$. From (2.22) and (2.23), $u$ is given as $\tilde{T} \rightarrow \infty$ by

$$
\begin{aligned}
\tilde{T}= & \ln u+C+O(u \ln u) \\
& +\delta\left[\frac{1}{2} \ln ^{2} u+(\bar{\kappa}-C) \ln u+D+O\left(u \ln ^{2} u\right)\right]+\cdots,
\end{aligned}
$$


and

$$
u=\exp [-\Phi / \delta], \quad \tilde{T}=\tilde{\widetilde{T}} / \delta .
$$

We choose $A$ and $B$ to match (2.39) and (2.40) with (2.41) in the intermediate limit $\widetilde{T} \gg 1, \widetilde{T} \ll 1$. Alternatively we match the expressions for $\delta \widetilde{T}$ and $\widetilde{\widetilde{T}}$ in the intermediate limit $u \ll 1, \Phi \ll 1$. Thus we introduce an intermediate variable $S$, defined by

$$
u=\exp [-S / \eta], \quad \Phi=\delta S / \eta, \quad \delta \ll \eta \ll 1,
$$

and consider the limit, $\eta, \delta / \eta \rightarrow 0$ with $S$ fixed (Cole 1968; Van Dyke, 1975).

We clearly require

$$
A=0
$$

then (2.43), (2.39) and (2.40) imply (eliminating $U$ )

$$
\tilde{\widetilde{T}} \sim \frac{\delta S}{\eta}-\delta B+O\left(\delta^{2}\right)
$$

From (2.43), $u \ln u$ and $u \ln ^{2} u$ are exponentially small terms in (2.41), therefore, this equation is

$$
\tilde{\widetilde{T}}=\delta \tilde{T} \sim \frac{\delta S}{\eta}+\delta C+O\left(\delta^{2}\right),
$$

and so matching stipulates that

$$
B=-C \text {. }
$$

To summarize, we have obtained the solution for $u$ implicitly in the two regions $\tilde{T} \sim 1, \tilde{T} \sim 1$ as follows (to leading order in $\delta$ ):

$$
\begin{aligned}
& \widetilde{T} \sim e^{-\check{\kappa} u} \int_{u}^{1} e^{\overline{\kappa \phi} \phi} \frac{d \phi}{\phi} \equiv \widetilde{T}_{0}(u), \quad \widetilde{T} \sim 1, \\
& u=\exp [-\Phi / \delta], \\
& \Phi \sim\left(U+\frac{1}{\bar{\kappa}}\right) \widetilde{T}_{0}(U)+\frac{1}{\bar{\kappa}} \ln U, \\
& \tilde{\widetilde{T}} \sim \widetilde{T}_{0}(U), \quad \tilde{T} \sim 1 .
\end{aligned}
$$

This solution represents the pulse of the periodic solution.

To complete the analysis of this section, we match the solution for $\tilde{\widetilde{T}} \sim 1$ into the dormant phase of the next pulse. If $g$ has period $P$ in $t$, then we must have

$$
u\left(\tilde{T} e^{\alpha P}\right)=u(\widetilde{T}) e^{-\alpha P} .
$$

Define

$$
T^{+}=\tilde{T} e^{-\alpha P}=\frac{\tilde{\widetilde{T}}}{\delta} e^{-\alpha P},
$$

so that (2.50) holds; i.e., $u(\tilde{T})=u\left(T^{+}\right) e^{-\alpha P}$. We determine $P$ by matching $u\left(T^{+}\right)$to 
$\phi(\tilde{\tilde{T}})$. To do so, introduce an intermediate variable $T_{\eta}$ via

$$
T^{+}=\eta T_{\eta}, \quad \tilde{\widetilde{T}}=\delta \eta e^{\alpha P} T_{\eta},
$$

where $\eta \ll 1$ (not the same $\eta$ as before) and $\delta \eta e^{\alpha . P} \gg 1$. From (2.49) and (2.22), $U \rightarrow 0$ exponentially as $\widetilde{\widetilde{T}} \rightarrow \infty$. Then $(2.35),(2.36),(2.22),(2.23),(2.44)$ and $(2.47)$ imply

$$
\Phi \sim \frac{C}{\bar{\kappa}}+\frac{\delta D}{\bar{\kappa}}+O\left(\delta^{2}\right)+\operatorname{TST}
$$

as $\eta \rightarrow 0, \delta \eta e^{\alpha P} \rightarrow \infty$, so that

$$
u \sim \exp \left[-\frac{1}{\delta}\left\{\frac{C}{\bar{\kappa}}+\frac{\delta D}{\bar{\kappa}}+O\left(\delta^{2}\right)\right\}\right] .
$$

But also

$$
u=u\left(T^{+}\right) e^{-\alpha P} \sim e^{-\alpha P}[1+O(\eta)] .
$$

We assume that $P$ is so large that $\eta$ can be chosen exponentially small in $\delta$. Then $O(\eta)$ is TST, and matching of (2.54) and (2.55) requires

$$
P=\frac{1}{\alpha \delta \bar{\kappa}}\left[C+\delta D+O\left(\delta^{2}\right)\right]
$$

to leading order, and recalling the definition of $C$ in (2.24), this is

$$
P=\frac{1}{\alpha \delta} \int_{0}^{1} e^{\bar{\kappa} u} \ln \left(\frac{1}{u}\right) d u+O\left(\frac{1}{\alpha}\right) .
$$

It follows that $\alpha P \sim 1 / \delta$, so that $\exp (-\alpha P)$ is transcendentally small, thus justifying the assumptions on $\eta$. The period grows almost exponentially with $\alpha$ : in Fig. 1 we plot the variation of $P$ with $\alpha$, for various values of $\bar{\kappa}$.

It is also of interest to calculate the maximum and minimum antigen quantities. When $\widetilde{T} \sim 1, g^{\prime}=0$ when $\widetilde{T} d u / d \widetilde{T}+u=0$, whence (2.17) implies

$$
\tilde{T}=e^{-\bar{\kappa} u} \int_{u}^{1} e^{\bar{\kappa} \phi} \frac{d \phi}{\phi}=\frac{1}{1-\bar{\kappa} u} ;
$$

solving this for $u$ gives $g_{\max }$ as

$$
g_{\max } \sim \frac{1}{\delta}\left[\frac{u}{1-\bar{\kappa} u}\right] .
$$

Thus $g_{\max } \sim O[\exp (\alpha)]$. Its variation with $\alpha$ is shown for various $\bar{\kappa}$ in Fig. 2 .

The minimum of $g$ occurs when $\tilde{\widetilde{T}} \sim 1, g=g_{\min }$ when $\tilde{\widetilde{T}} \phi^{\prime}(\widetilde{\widetilde{T}})=\delta$. From $(2.30)$ $\phi^{\prime}=U+O(\delta)$, where $\widetilde{\widetilde{T}} \sim T_{0}(U)+O(\delta) ;$ thus $g=g_{\min }$ when

$$
U \tilde{T}_{0}(U)=\delta \text {, }
$$

and at this point (2.37) implies

$$
\phi \sim \delta\left[1+\frac{1}{\bar{\kappa} U}\right]+\frac{1}{\bar{\kappa}} \ln U .
$$


Fig. 1. Variation of the period $P$ with $\alpha$, for values of $\bar{\kappa}=0.01,0.1,1.0$, from the asymptotic estimate (2.57)

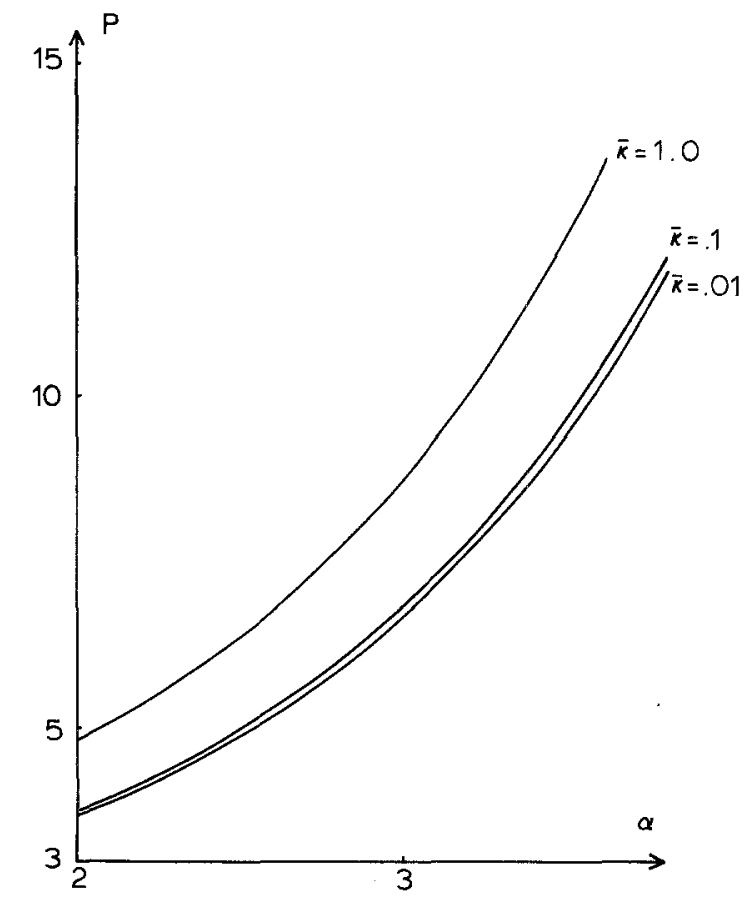

Fig. 2. Variation of $g_{\max }$ with $\alpha$, for values of $\bar{\kappa}=0.01,0.1,1.0$, from solving (2.58) and (2.59)

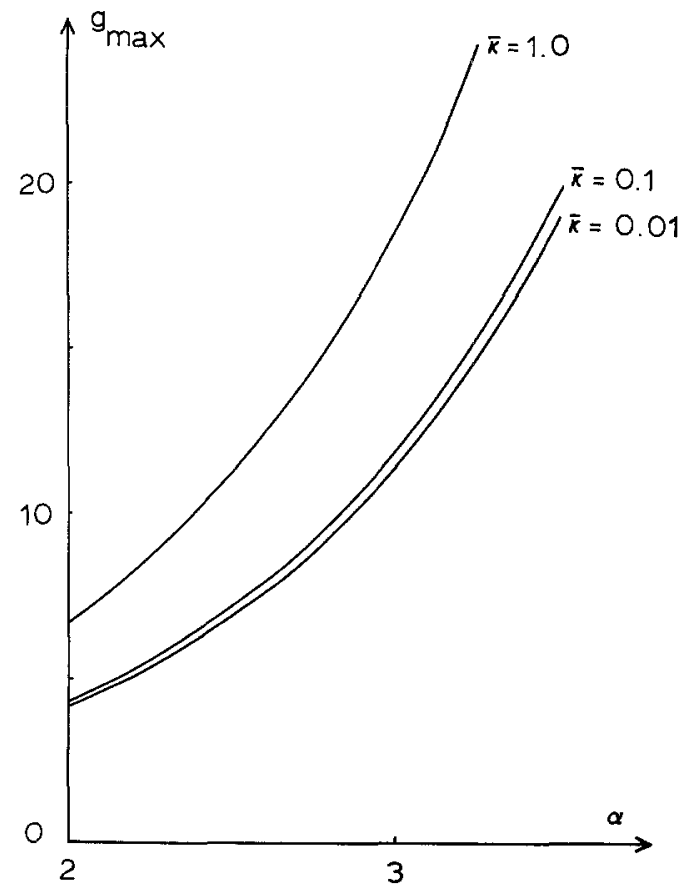




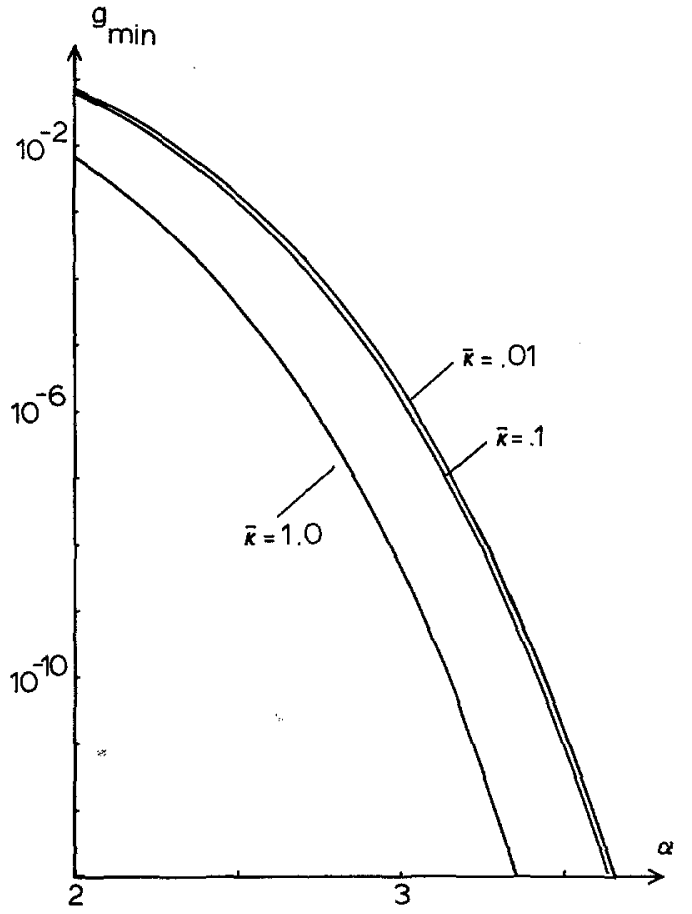

Fig. 3. Variation of $g_{\min }$ with $\alpha$, for values of $\bar{\kappa}=0.01,0.1,1.0$, from the asymptotic estimate (2.63)

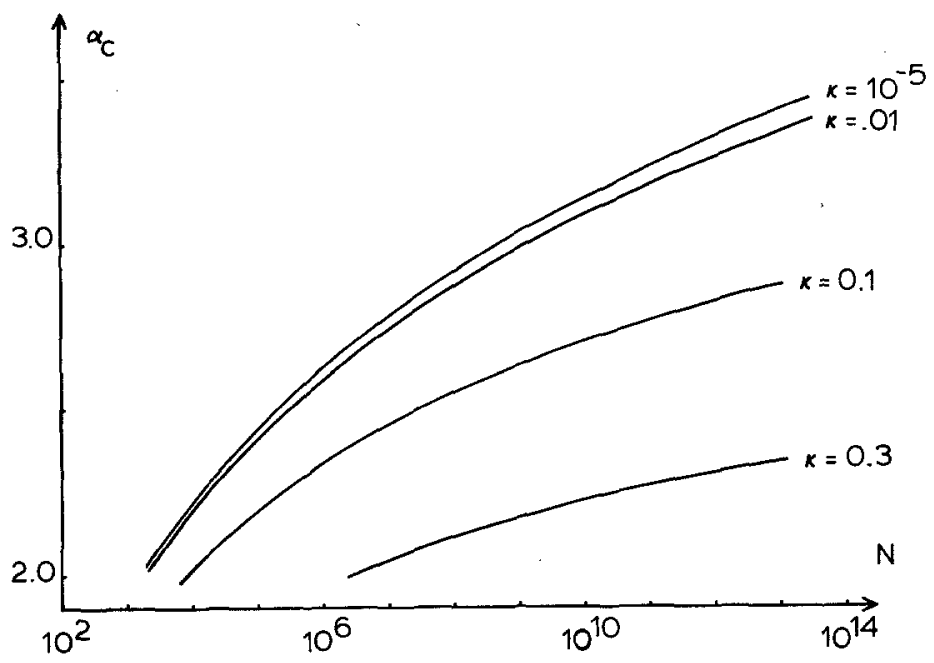

Fig. 4. Variation of $\alpha_{c}$ with $N$ for various $\kappa$, from (2.64). Even for large $N$, the critical value of $\alpha$ is very low (for all values of $\kappa$ )

We can gain an explicit estimate by using the smallness of $\delta$ to solve (2.60). Using the asymptotic description of $\widetilde{T}_{0}$ as $U \rightarrow 0$, we find

$$
\phi \sim \frac{C}{\bar{\kappa}}+O(\delta)
$$


whence eventually

$$
g_{\min } \sim \alpha \exp \left[2 \alpha-e^{\alpha} \int_{0}^{1} e^{\bar{k} u} \ln \left(\frac{1}{u}\right) d u\right] .
$$

Of course $g_{\min }$ is exponentially small as $\alpha \rightarrow \infty$. Increasing $\bar{\kappa}$ decreases $g_{\min }$ even further, as shown in Fig. 3, where we plot $g_{\min }$ versus $\alpha$ for different $\bar{\kappa}$ values.

If $g_{s t}=N$ cells (typically we might expect $N \sim 10^{3}$ or $10^{4}$ ), then the critical delay when elimination by stochastic means becomes possible is when $g_{\min } \sim 1 / N$. The exponential exponent is the dominant term in (2.63), and a reasonable leading order estimate for the critical value of $\alpha$ is thus

$$
\alpha_{c} \sim \ln \left[\frac{\ln N}{\int_{0}^{1} e^{\bar{k} u} \ln (1 / u) d u}\right] .
$$

This defines the critical delay $T_{c}$ via $\alpha_{c}=K T_{c}$. Since $\alpha_{c} \sim \ln \ln N$ as $N$ becomes large, $\alpha$ need not itself be very large at all to effect complete extinction. In Fig. 4 we plot the variation of $\alpha_{c}$ with $N$ for various values of $\kappa($ not $\bar{\kappa})$.

\section{Approach to Criticality}

The results of the previous section would be sufficient for most practical purposes. However, it is known that the periodic oscillation ceases to exist if $\kappa$ and hence $\bar{\kappa}$ are sufficiently large. In fact, the requirement (2.12) that $\kappa$ should be $O(\delta)$ in order to obtain a "correct" scaling should automatically warn us that some kind of criticality behaviour is to be expected. (1.12) gives Dibrov's et al. (1977b) criticality condition for non-existence of a periodic solution as $\kappa>\alpha \exp (1-\alpha)$ : in terms of $\delta$ and $\bar{\kappa}$, this is

$$
\bar{\kappa}>e \ln \left(\frac{1}{\delta}\right) .
$$

In this section we re-examine the asymptotic procedure of $\$ 3$ in order to study this criticality.

We first study the asymptotic results of the preceding section when $\bar{\kappa}$ becomes large. Evaluating $\widetilde{T}_{0}$ given by (2.20) by Laplace's method (Murray, 1974) shows that, as $\bar{\kappa} \rightarrow \infty$,

$$
\widetilde{T}_{0}=e^{-\bar{\kappa} u} \int_{u}^{1} e^{\bar{\kappa} \phi} \frac{d \phi}{\phi} \sim \frac{1}{\bar{\kappa}} e^{\bar{\kappa}(1-u)} ;
$$

Thus $\widetilde{T}_{0} \sim 1 / \delta$ when $\bar{\kappa} \sim \ln (1 / \delta)$, and this suggests a new asymptotic analysis in this case. To study this, we define

$$
\bar{\kappa}=\frac{1}{\Delta} \ln \left(\frac{1}{\delta}\right), \quad \Delta \sim 1,
$$

so that

$$
\delta=\exp [-\bar{\kappa} \Delta], \quad \bar{\kappa} \gg 1 ;
$$

thus $\delta$ is exponentially small compared to any power of $1 / \bar{\kappa}$. 
Now (2.13) is

$$
\frac{d u}{d \widetilde{T}}=\frac{-u u(\delta \tilde{T})}{1-\delta \bar{\kappa}+\bar{\kappa} \widetilde{T} u}, \quad u(0)=1
$$

Since $\bar{\kappa} \gg 1$, this suggests a time scale $\widetilde{T} \sim 1 / \bar{\kappa}$; we therefore put

$$
\tau=\bar{\kappa} \tilde{T}
$$

so that

$$
\frac{d u}{d \tau}=\frac{-u u(\delta \tau)}{\bar{\kappa}[1-\delta \bar{\kappa}+\tau u]}
$$

we now solve (4.7) with $u(0)=1$ as a power series in $1 / \bar{\kappa}$, neglecting terms of $O(\delta)$, since this is transcendentally small by comparison. The result is

$$
u \sim 1-\frac{1}{\bar{\kappa}} \ln (1+\tau)+\frac{1}{\bar{\kappa}^{2}}\left[\frac{\tau-\ln (1+\tau)}{1+\tau}\right] \cdots .
$$

This series is the analogue of (2.14). Since $g=\tilde{T} u / \delta$, we require $u$ to decrease (towards zero) if we are to obtain a periodic solution. If $u$ decreases too slowly [as suggested by (2.11) if $\kappa \sim 1$ ] then $g$ will grow uncontrollably. Thus we expect the critical behaviour to be associated with a slowing down of the rate of decrease of $u$, as evidenced by (4.8).

The series (4.8) fails when $\ln \tau \sim \bar{\kappa}$; this suggests a new time defined by

$$
\tau=\exp [\bar{\kappa} \tilde{\tau}], \quad u(\tau) \equiv \tilde{u}(\tilde{\tau})
$$

using (4.9), we find $u(\delta \tau) \equiv \tilde{u}(\tilde{\tau}-\Delta)[\Delta$ given by (4.3)], and (4.7) becomes

$$
\exp [-\bar{\kappa} \tilde{\tau}][1-\delta \bar{\kappa}] \frac{1}{\tilde{u}} \frac{d \tilde{u}}{d \tilde{\tau}}+\frac{d \tilde{u}}{d \tilde{\tau}}=-\tilde{u}(\tilde{\tau}-\Delta) \text {. }
$$

This equation is valid when $\tilde{\tau} \sim 1$ : it is invalid when $\tau \sim 1$, i.e., $\tilde{\tau} \sim 1 / \bar{\kappa}$. The righthand side $(=-u(\delta \tau))$ can therefore only be used in the form shown when $\tilde{\tau}-\Delta \sim 1(\gg 1 / \bar{\kappa})$. When $\tilde{\tau}-\Delta \lesssim 1 / \bar{\kappa}$, then it must be written in the form $-u(\delta \tau)$. Now for $\tilde{\tau}-\Delta<0(|\tilde{\tau}-\Delta| \gg 1 / \bar{\kappa}),(4.9)$ gives $\delta \tau=\exp [\bar{\kappa}(\tilde{\tau}-\Delta)]$, which is exponentially small in $1 / \bar{\kappa}$; thus $(4.8)$ implies $u(\delta \tau)=1+\exp [-O(\bar{\kappa})]$ there. In the region $|\tilde{\tau}-\Delta| \sim 1 / \bar{\kappa}$, the right-hand side of $(4.10)$ must be replaced by the appropriate form of (4.8).

Now let $\bar{\kappa} \rightarrow \infty$ for fixed $\tilde{\tau}$. As long as $\tilde{u} \sim 1(>0)$, the first term in (4.10) is exponentially small for all $\tilde{\tau}>0$. It follows from the above remarks that $\tilde{u}$ satisfies, at leading order in $1 / \bar{\kappa}$,

$$
\begin{aligned}
\frac{d \tilde{u}}{d \tilde{\tau}} & =-\tilde{u}(\tilde{\tau}-\Delta), \quad \tilde{\tau}>\Delta, \\
\frac{d \tilde{u}}{d \tilde{\tau}} & =-1, \quad 0<\tilde{\tau}<\Delta, \\
\tilde{u}(0) & =1 .
\end{aligned}
$$


$O(1 / \bar{\kappa})$ corrections arise from $\tilde{\tau}$ near $\Delta$ (and hence $2 \Delta$, etc.) and also from matching $\tilde{u}$ to (4.8) as $\tau \rightarrow \infty, \tilde{\tau} \rightarrow 0$. (4.11) is equivalent to the linear delay equation

$$
\begin{aligned}
\frac{d \tilde{u}}{d \tilde{\tau}} & =-\tilde{u}(\tilde{\tau}-\Delta), \quad \tilde{\tau}>0, \\
\tilde{u} & =1, \quad \tilde{\tau} \in(-\Delta, 0] .
\end{aligned}
$$

This admits solutions of the form

$$
\tilde{u}=\exp [-\gamma \tilde{\tau}], \quad \gamma=e^{\gamma \Delta}
$$

for real $\gamma \Delta, \gamma \Delta e^{-\gamma \Delta}<e^{-1}$, hence if $\Delta>e^{-1}$, all solutions of the form (4.13) are oscillatory, whereas if $\Delta<e^{-1}$, two real values of $\gamma$ exist. In fact, it is known (Driver, 1976; Myshkis, 1955) that for (4.12), $\tilde{u}>0$ for all $\tilde{\tau}$ if $\Delta<e^{-1}$, whereas if $\Delta>e^{-1}$, there exists $\tilde{\tau}^{*}$ such that $\tilde{u}\left(\tilde{\tau}^{*}\right)=0, \tilde{u}>0$ for all $\tilde{\tau}<\tilde{\tau}^{*}$, and $\tilde{u}$ approaches zero oscillatorily (if $\Delta<\pi / 2$ ).

This distinction is exactly the critical behaviour we seek. For if $\tilde{u} \rightarrow 0$ at finite $\tilde{\tau}$, then certainly $g \rightarrow 0$ there: thus a pulse exists. However, the first term in (4.10) then becomes relevant ( since $\tilde{u} \rightarrow 0$ ), and we suppose that the solution continues to grow again. If, on the other hand, $\Delta<e^{-1}$, then $\tilde{u}>0$ for all $\tilde{\tau}, \tilde{u} \sim \exp [-\gamma \tilde{\tau}]$ as $\tilde{\tau} \rightarrow \infty$, where $\gamma$ is the smaller root of $\gamma=\exp (\gamma \Delta),(1 / \tilde{u}) d \tilde{u} / d \tilde{\tau}$ is bounded as $\tilde{u} \rightarrow 0$, and so (4.12) provides a uniformly accurate approximation to (4.10) for all $\tilde{\tau}>0$. To establish what this entails for $g$ we recollect the definition of $\tilde{\tau}$. We have $\tau=\exp (\bar{\kappa} \tilde{\tau})$, $\tau=\bar{\kappa} \widetilde{T}, T=\tilde{T} / \delta, T=\exp (\alpha t), \delta=\exp (-\alpha), \bar{\kappa}=(\ln (1 / \delta)) / \Delta ;$ also $g=\tilde{T} \tilde{u} / \delta . A$ little arithmetic shows

$$
\begin{aligned}
& \tilde{\tau}=\frac{1}{\bar{\kappa}} \ln \bar{\kappa}+\Delta(t-1), \\
& g \sim \exp \left[-\frac{\gamma}{\bar{\kappa}} \ln \bar{\kappa}+\gamma \Delta+(\alpha-\gamma \Delta) t\right] .
\end{aligned}
$$

Since $\gamma \Delta<1, g$ grows exponentially with time provided $\alpha>1$, (which is a necessary condition for instability of the steady state). Thus, at leading order in $1 / \bar{\kappa}$, we obtain a condition that no oscillation exists if $\Delta<e^{-1}$. In terms of $\bar{\kappa}$, this is, from (4.3), $\bar{\kappa}>e \ln (1 / \delta)$, and is identical to the exact criterion (4.1), even though apparently only a leading-order approximation to it. This shows the power of the asymptotic approach.

No explicit description of the periodic solution when $e^{-1}<\Delta \sim O(1)$ seems feasible. However, based on the method of steps (Driver, 1975), we are able to carry out a partial analysis. We find, for example, that $\tilde{\tau}^{*}$ (given by $\tilde{u}\left(\tilde{\tau}^{*}\right)=0$ ) satisfies

$$
\begin{aligned}
& \tilde{\tau}^{*}=1, \quad \Delta>1, \quad\left(\tilde{\tau}^{*} \in(0, \Delta)\right), \\
& \tilde{\tau}^{*}=1+\Delta-\{2 \Delta-1\}^{1 / 2}, \quad 2-\sqrt{2}<\Delta<1 \quad\left(\tilde{\tau}^{*} \in(\Delta, 2 \Delta)\right),
\end{aligned}
$$

etc. If $t=t^{*}$ when $\tilde{\tau}=\tilde{\tau}^{*},(4.14)$ gives

$$
t^{*}=1+\frac{1}{\Delta}\left[\tilde{\tau}^{*}-\frac{1}{\bar{\kappa}} \ln \bar{\kappa}\right] \approx 1+\tilde{\tau}^{*} / \Delta
$$


for large $\bar{\kappa} .(4.15)$ and (4.16) show that as $\bar{\kappa}$ increases (so $\Delta$ decreases), $t^{*}$ increases towards infinity. $t^{*}$ is a measure of how long the pulse lasts: thus as criticality is approached, the outbreak becomes longer and longer. The maximum of $g$ may also be estimated from (4.12), and is roughly

$$
g_{\max } \sim \exp \left(\alpha t^{*}\right) .
$$

We may correspondingly estimate the succeeding minimum of $g$ as $g_{\min } \sim$ $\exp \left[-g_{\max }\right]$, by analogy with $\S 3$, and hence also the period as $P \sim g_{\max } / \alpha$. Thus as $\bar{\kappa}$ increases, the period of the oscillation and maximum value of $g$ both increase exponentially with the outbreak (pulse) duration, and the minimum descreases doubly exponentially with it.

\section{Discussion and Conclusions}

In this paper we have sought analytic solutions for the antigen population $g$ and antibody population $a$ in a simplistic model proposed by Dibrov et al. (1977b) in which the small $B$ cell population remains constant, and the delay in cell maturation after antigen stimulus is taken into account.

When this model is formulated in dimensionless terms, there are three dimensionless parameters which naturally arise; one of these, $\alpha$, measures the ratio of the maturation delay to the growth time of the infecting antigen. When $\alpha>\pi / 2$, the non-trivial steady state in which non-zero antibody and antigen populations can coexist is always linearly unstable, and provided another parameter $\kappa$, which essentially measures the antigen growth rate, is small enough $\left(\kappa<\alpha e^{1-\alpha}\right)$, there exists a steady periodic solution which, for reasonable values of $\alpha$, has the appearance (in both $a$ and $g$ ) of a series of spiky pulses separated by longer periods of dormant activity (low levels of $a$ and $g$ ). The pulses in the controlling antibody $a$ occur slightly after the antigen pulses.

We have shown how an asymptotic method based on the limit of large delay $(\alpha \rightarrow \infty)$ is able to predict the observed form of these oscillations. The power of the method, which consists of solving the equations asymptotically in terms of an exponential time scale $\exp (\alpha t)$, lies in the fact that quite accurate estimates can be obtained even for very moderate values of $\alpha$. This is because the small parameter which occurs is really $\exp (-\alpha)$. Further, the minimum value of antigen $g$ in the oscillation is $\exp [-O(\exp \alpha)]$, which gives the observed flat phase in between spikes for $\alpha$ as low as 1.5. This last quantity is of some use in the present context, because very low minima in the oscillation lead to the possibility that the infecting antigen may be completely eliminated, since when antigen quantities approach unity (deterministically), realistic stochastic processes predict that the probability of extinction becomes $O(1)$.

The analysis proceeds on the basis that $\alpha \gg 1, \beta / \alpha \rightarrow \infty, \kappa \sim e^{-\alpha}=\delta$ (the last being a necessary condition as $\alpha \rightarrow \infty$ ). Through a variety of transformations, the equations are manipulated to a form where they can be solved approximately. Asymptotic expansions for the values of $g_{\max }, g_{\min }$ and $P$ (the period) can then be obtained. To leading order, these are

$$
g_{\max } \sim O\left(e^{\alpha}\right)
$$




$$
g_{\min } \sim \alpha \exp \left[2 \alpha-e^{\alpha} \int_{0}^{1} e^{\bar{\kappa} u} \ln \frac{1}{u} d u\right]
$$

where

$$
\bar{\kappa}=\kappa e^{\alpha} \sim 1,
$$

and

$$
P=\frac{e^{\alpha}}{\alpha} \int_{0}^{1} e^{\bar{\kappa} u} \ln \frac{1}{u} d u+O\left(\frac{1}{\alpha}\right) .
$$

For $\bar{\kappa}=0$, these concur with the results of Fowler (1981).

The deterministic model predicts that for $\alpha$ greater than a critical value [this is $\left(\cos ^{-1} \kappa\right) /\left(1-\kappa^{2}\right)^{1 / 2}$ if $\left.\beta \rightarrow \infty\right]$, there exists a periodic oscillation which disappears when $\alpha>M$, where $M e^{1-M}=\kappa$. For $\alpha>M$, the antigen $g$ grows indefinitely. However, there exists a critical delay $T_{c}$ (or critical $\alpha_{c}$ ) such that if $\alpha>\alpha_{c}$, the minimum phase of the infection is so low that complete elimination is virtually assured. If $N$ antigen cells can be supported in the steady state in the absence of any cell maturation delay, then the critical value of $\alpha$ is when $g_{\min } \sim 1 / N$. For $\alpha \gg 1$, this gives approximately

$$
\alpha_{c} \sim \ln \ln N
$$

so that quite moderate delays can help to eliminate antigen entirely. There is thus a "barrier" region $\alpha_{c}<\alpha<M$ within which the animal's immune response can counteract any infection by removing it entirely. For $\alpha<\alpha_{c}$, oscillating antigen populations can be maintained with a maximum antigen colony of $\sim N \ln N$ cells [from (5.1)], and a maximum period of $T \ln N\left(\sim 1\right.$ month if $T \sim 4$ days, $N \sim 10^{3}$ cells), which is not too contagious. However, for $\alpha$ above $M$, the antigen is too virulent and cannot be controlled at all. There are thus three regimes: sub-critical, when a permanent antigen population ( $N$ cells if stable, maximum $\sim N \ln N$ cells if oscillatory) can be tolerated; a "barrier", when any infection is almost certainly eliminated; and a supercritical regime, where any infection is uncontrollable.

The above results remain qualitatively valid when $\bar{\kappa}$ becomes large, but the analysis has to be adjusted when $\bar{\kappa}=O(\alpha)$. One finds that if $\bar{\kappa}>\alpha e$, then the supercritical state always occurs. If $\bar{\kappa}<\alpha e$, then the previous description applies, but the nature of the solution changes. If $t^{*}$ is the duration of the pulse in units of the maturation delay time, then when $\bar{\kappa} \sim 1, t^{*} \approx 1$, whereas as $\bar{\kappa}$ increases towards $\alpha e$, $t^{*}$ increases towards infinity, and

$$
g_{\max }=O\left(e^{\alpha t^{*}}\right) .
$$

We would expect the period $P$ to correspondingly increase, and the minimum $g_{\text {min }}$ to decrease, but the analytic results for these variables are not available.

It is instructive to consider the effect on $\bar{\kappa}$ and $\alpha$ of varying the physical parameters $K$ (antigen growth rate) and $T$ (maturation delay) separately. In either case, the dynamics must successively pass through the sub-critical, barrier, and supercritical regimes. This is certainly true if the delay $T$ is increased with constant $K$, and is also true if $K$ (antigen virulence) is increased at constant $T$, provided the delay is sufficiently large, in fact if $T>R / A Q$, as is easily ascertained 
by an examination of Fig. 7 of Dibrov et al. (1977b). If $T<R / A Q$, then the response switches from subcritical to supercritical as $K$ increases through $1 / T$.

\section{References}

Banks, H. T.: Delay systems in biological models: Approximation techniques. In: Nonlinear systems and applications, (V. Lakshmikantham, ed.) pp. 21-38. New York: Academic Press 1977

Bell, G. I.: Mathematical model of clonal selection and antibody production. J. Theor. Biol. 29, $191-232(1970)$

Bell, G. I.: Mathematical model of clonal selection and antibody production. II. J. Theor. Biol. 33, $339-378(1971)$

Bell, G. I.: Predator-prey equations simulating an immune response. Math. Biosci. 16, $291-314$ (1973)

Cole, J. D.: Perturbation methods in applied mathematics. Waltham, MA: Blaisdell 1968

Cushing, J. M. : Integro-differential equations and delay models in population dynamics. Lecture Notes in Biomathematics, Vol. 20. Berlin: Springer 1978

Dibrov, B. F., Livshits, M. A., Volkenstein, M. V.: Mathematical model of immune processes. J. Theor. Biol. 65, 609-631 (1977a)

Dibrov, B. F., Livshits, M. A., Volkenstein, M. V.: Mathematical model of immune processes. II. Kinetic features of antigen-antibody interrelations. J. Theor. Biol. 69, 23-39 (1977b)

Driver, R. D.: Ordinary and delay differential equations. Berlin: Springer 1975

Driver, R. D.: Linear differential systems with small delays. J. Diff. Equat. 21, 148-166 (1976)

Eisen, H. N.: Immunology. Hagerstown, MD: Harper \& Row 1974

Fowler, A. C.: An asymptotic analysis of the logistic delay equation. J. Inst. Maths. Applics., to appear (1981)

Hood, L. E., Weissmann, I. L., Wood, W. B.: Immunology. Menlo Park, CA: Benjamin/Cummings 1978

Jones, G. S.: On the nonlinear differential-difference equation $f^{\prime}(x)=-\alpha f(x-1)\{1+f(x)\}$. J. Math. Anal. Appl. 4, 440-469 (1962a)

Jones, G. S.: The existence of periodic solutions of $f^{\prime}(x)=-\alpha f(x-1)\{1+f(x)\}$. J. Math. Anal. Appl. 5, 435-450 (1962b)

Li, T. V., Yorke, J. A.: Period three implies chaos. Amer. Math. Monthly 82, 985-992 (1975)

Lorenz, E. N.: Deterministic non-periodic flow. J. Atmos. Sci. 20, 130-141 (1962)

Ludwig, D., Jones, D. D., Holling, C. S.: Qualitative analysis of insect outbreak systems: The spruce budworm and the forest. J. Anim. Ecol. 47, 315-332 (1978)

Macdonald, N.: Times lags in biological models. Lecture notes in biomathematics, Vol. 27. Berlin: Springer 1978

Mackey, M. C., Glass, L.: Oscillation and chaos in physiological control systems. Science 197, 287-289 (1977)

Merrill, S. J.: A model of the stimulation of B-cells by replicating antigen-I and II. Math. Biosci. 41, $125-141$ and $143-155(1978 \mathrm{a}, \mathrm{b})$

Morris, H. C.: A perturbative approach to periodic solutions of delay-differential equations. J. Inst. Maths. Applics. 18, 15-24 (1976)

Murray, J. D.: Asymptotic analysis. Oxford: Clarendon Press 1974.

Myshkis, A. D.: Linear differential equations with a retarded argument. (German translation). Berlin: VEB Deutscher Verlag 1955

Nye, J. F.: Water flow in glaciers: jökulhlaups, tunnels, and veins, J. Glaciol. 17, $181-207$ (1976)

Perelson, A. S., Mirmirani, M., Oster, G. F.: Optimal strategies in immunology, I. B-cell differentiation and proliferation. J. Math. Biol. 3, 325-367 (1976)

Perelson, A. S., Mirmirani, M., Oster, G. F.: Optimal strategies in immunology II: B memory cell production. J. Math. Biol. 5, 213-256 (1978)

Pimbley, G. H.: Periodic solutions of third order predator-prey equations simulating an immune response. Arch. Rat. Mech. Anal. 55, $93-124$ (1974)

Pimbley, G. H.: Bifurcation behaviour of periodic solutions for an immune response problem. Arch. Rat. Mech. Anal. 64, 169-192 (1977)

Romball, C. G., Weigle, W. O. : A cyclical appearance of antibody-producing cells after a single injection of serum protein antigen. J. Exp. Med. 138, 1426-1442 (1973) 
Rubinow, S. I. : Age-structured equations in the theory of cell populations. In: Studies of mathematical biology, Part II : Populations and communities. (S. A. Levin, ed.) MAA studies in mathematics, Vol. 16. Washington, D. C.: Mathematical Association of America 1978

Spiegelberg, H. L.: Biological activities of immunoglobulins of different classes and sub-classes. Adv. Immunol. 19, 256-294 (1974)

Tew, J. G., Mandel, T. : The maintenance and regulation of serum antibody levels: Evidence indicating a role for antigen retained in lymphoid follicles. J. Immunol. 120, 1063-1069 (1978)

Van Dyke, M. D.: Perturbation methods in fluid mechanics (2nd ed.). Stanford, CA: Parabolic Press 1975

Waltman, P., Butz, E.: A threshold model of antigen-antibody dynamics. J. Theor. Biol. 65, 499-512 (1977)

Weigle, W. O.: Cyclical production of antibody as a regulatory mechanism in the immune response. Adv. Immunol. 21, 87-111 (1975)

Received March 13, 1980/Revised March 27, 1981 